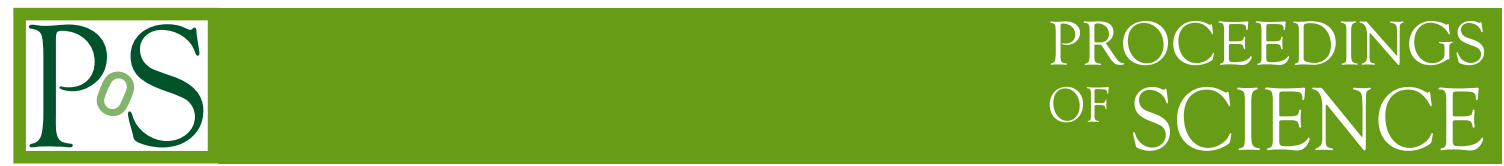

\title{
Chiral perturbation theory tests.
}

\author{
Riccardo Fantechi ${ }^{* \dagger}$ \\ INFN Pisa, Italy \\ E-mail: riccardo.fantechi@pi.infn.it
}

The NA48/2 and NA62 experiment have a long tradition in the study of decay modes of charged kaons, with the goal to check the ChPT predictions. This paper will show the latest results for $\mathrm{K}^{ \pm} \rightarrow \pi^{ \pm} \gamma \gamma$ and a progress report on $\mathrm{K}^{ \pm} \rightarrow \mathrm{e}^{ \pm} v \gamma$ and $\mathrm{K}^{ \pm} \rightarrow \pi^{ \pm} \pi^{0} e^{+} e^{-}$.

The XIth International Conference on Heavy Quarks and Leptons,

June 11-15, 2012

Prague, Czech Republic

\footnotetext{
*Speaker.

$\dagger$ on behalf of the NA48/2 and NA62 Collaborations.
} 


\section{Introduction}

During 2003 and 2004, the NA48/2 experiment has collected at the CERN SPS the world largest amount of charged kaon decays, with the main goal to search for direct $\mathrm{CP}$ violation in the decay of $\mathrm{K}^{ \pm}$into three pions [1]. Later, in 2007/2008, the NA62 experiment has taken data, using the same detector and a slightly modified beam, to measure the ratio of the decay rates of the $K \rightarrow e v$ and $K \rightarrow \mu \nu$ decays. The large statistics accumulated in those runs has allowed, exploiting minimum bias and downscaled control triggers, the study of many rare charged kaon decays. We report here the latest results for the decays $\mathrm{K}^{ \pm} \rightarrow \pi^{ \pm} \gamma \gamma$ and the prospects for $\mathrm{K}^{ \pm} \rightarrow \mathrm{e}^{ \pm} v \gamma$ and $\mathrm{K}^{ \pm} \rightarrow \pi^{ \pm} \pi^{0} e^{+} e^{-}$, together with the predictions of Chiral Perturbation Theory [2]. ChPT is a powerful framework to provide high precision predictions for the mesonic sector, and in particular for K decays.

\section{The NA48/2 beam}

The beam line used in the NA48/2 [1] data taking was specifically designed to transport simultaneously positive and negative particles with a central momentum of $60 \mathrm{GeV} / \mathrm{c}$. The positive and negative beams were produced with $400 \mathrm{GeV} / \mathrm{c}$ primary protons from the SPS accelerator with a nominal intensity on target of $7 \cdot 10^{11}$ protons/spill impinging on a beryllium target of $40 \mathrm{~cm}$ length and $2 \mathrm{~mm}$ diameter at zero incidence angle. A central momentum and momentum bite of $60 \pm 3 \mathrm{GeV} / \mathrm{c}$ was selected symmetrically for both positively and negatively charged particles by their passage through achromat systems, namely two vertical deflection magnets with opposite-sign field and a pair of dump collimators with variable but similar openings for each of the two beams. Following these dump collimators, which also served to absorb the remaining primary protons, two further vertical deflection magnets returned the beams onto a common axis. The beams were selected with the same geometrical acceptance and directed along a common line. Both beams were focused to similar and small spot sizes $(\approx 5 \mathrm{~mm} \mathrm{r.m.s.})$ at the spectrometer position, so as to minimize the transverse scale of any structure within the beam spots. During 2004 data taking, the positive beam flux at the exit of the final collimator was estimated to be $3.8 \cdot 10^{7}$ particles per pulse (ppp) (of which $2.2 \cdot 10^{6}$ were $^{+}$). The negative beam flux was $2.6 \cdot 10^{7} \mathrm{ppp}\left(1.3 \cdot 10^{6}\right.$ ). The fraction of kaon decays in the decay volume was $22 \%$.

\section{The NA62 beam}

The beam line and setup of the earlier NA48/2 experiment were used for the NA62 data taking in 2007 [3]. The momentum of the unseparated secondary positive and/or negative hadronic beams was chosen to be $74 \mathrm{GeV} / \mathrm{c}$ with a spread of $\pm 1.4 \mathrm{GeV} / \mathrm{c}$ (rms). The reduction of the momentum spread was achieved with a tighter collimation scheme, partially compensated by an increase in the proton beam intensity. With $1.8 \cdot 10^{12}$ protons/spill on target, the positive secondary beam flux at the entrance of the decay volume is $2.5 \cdot 10^{7} \mathrm{ppp}$, with a kaon fraction of $6 \%$. The fraction of kaon decays in the decay volume was $18 \%$. The configuration of the muon scrapers was changed, with the side effect of a charge-asymmetric muon halo background. For this reason, most of the data taking was recorded with the $\mathrm{K}^{+}$beam only. 


\section{The NA48/2 and NA62 detector}

The momenta of charged decay products were measured by a magnetic spectrometer, housed in a tank filled with helium at nearly atmospheric pressure and placed after the decay volume. The spectrometer accomodated four drift chambers composed each of 8 planes of sense wires, and a dipole magnet located between the second and the third DCHs, which gave a horizontal transverse momentum kick to charged particles of respectively $120 \mathrm{MeV} / \mathrm{c}$ in 2004 and $265 \mathrm{MeV} / \mathrm{c}$ in 2007 . The corresponding measured spectrometer momentum resolution were $\sigma_{p} / p=1.02 \% \oplus 0.044 \% p$ in 2004 and $\sigma_{p} / p=0.48 \% \oplus 0.009 \% p$ in 2007, where the momentum $p$ is expressed in GeV/c. A counter hodoscope consisting of two planes of orthogonal plastic scintillator strips producing fast trigger signals was placed after the spectrometer. A $127 \mathrm{~cm}\left(27 X_{0}\right)$ thick liquid krypton electromagnetic calorimeter located further downstream is used to measure energy and position of photons and electrons. Its 13248 readout cells had a transverse size of $2 \times 2 \mathrm{~cm}^{2}$ each without longitudinal segmentation. The energy resolution was $\sigma_{E} / E=3.2 \% / \sqrt{E} \oplus 9 \% / E \oplus 0.42 \%$ (E in $\mathrm{GeV}$ ). The spatial resolution for the transverse coordinates $\mathrm{x}$ and $\mathrm{y}$ of an isolated electromagnetic shower was $\sigma_{x}=\sigma_{y}=0.42 / \sqrt{E} \oplus 0.06 \mathrm{~cm}(\mathrm{E}$ in $\mathrm{GeV})$. A description of the detector can be found in [4].

The very good performances of the liquid krypton calorimeter are of paramount importance to reduce the background levels in all the analyses described here.

\section{Experimental results}

\section{$5.1 \mathrm{~K}^{ \pm} \rightarrow \pi^{ \pm} \gamma \gamma$}

The decay $\mathrm{K}^{ \pm} \rightarrow \pi^{ \pm} \gamma \gamma$ has no tree $\mathrm{O}\left(\mathrm{p}^{2}\right)$ contribution; the leading contribution is at one-loop order $\mathrm{O}\left(\mathrm{p}^{4}\right)$ with an undetermined constant $\hat{\mathrm{c}}$ of order 1 [5](Fig. 1-a).

$\mathrm{O}\left(\mathrm{p}^{6}\right)$ high order corrections to the branching ratio are expected to be about $30-40 \%$. In addition, corrections from $K \rightarrow 3 \pi$ give rise to a y-dependence, where $y=P_{K}\left(P_{\gamma 1}-P_{\gamma 2}\right) / M_{K}^{2}$ and a non-zero rate for $M_{\gamma \gamma} \rightarrow 0$ (Fig. 1-b). Both $\mathrm{O}\left(p^{4}\right)$ and $\mathrm{O}\left(p^{6}\right)$ predictions show in the z-spectrum (where $\left.z=\left(M_{\gamma \gamma} / M_{K}\right)^{2}\right)$ a cusp-like structure at a $\mathrm{z}$ value corresponding to the $2 m_{\pi^{+}}$mass. Fig. 1-c shows the branching ratio as a function of $\hat{c}$ in the two predictions. The E787 experiment at BNL observed this decay for the first time [6], with 31 events and an estimated background of $5.1 \pm 3.3$ events in the range $100 \mathrm{MeV} / \mathrm{c}<P_{\pi}<180 \mathrm{MeV} / \mathrm{c}$ and a corresponding partial branching ratio of $\left(6.0 \pm 1.5_{\text {stat }} \pm 0.7_{\text {syst }}\right) \cdot 10^{-7}$.

The analysis of this decay has been done using data from NA48/2 (run 2004) and from NA62 (run 2007). The results are first shown separately and then their combination is presented. NA48/2 data were collected during a 3-day minimum bias run in 2004. The NA62 data used come from a set of control triggers, downscaled by an average factor of 20 during the 3 months data taking for the $R_{K}$ measurement[3]. The ratio of fluxes is $\Phi(2004) / \Phi(2007)=0.7$ and the two data sets have different acceptances and similar systematics, taken into account in the final combination.

Signal events were selected in the region $z>0.2$ to minimize the background from $\pi^{+} \pi^{0}(\gamma)$. The normalization channel is $\mathrm{K}^{ \pm} \rightarrow \pi^{ \pm} \pi^{0}$ with a selection $\left|M_{\gamma \gamma}-M_{\pi^{0}}\right|<10 \mathrm{MeV} / \mathrm{c}^{2}$. Mass resolutions are respectively $\sigma_{\pi \gamma \gamma}=5.9 \mathrm{MeV} / \mathrm{c}^{2}$ and $\sigma_{\pi \pi^{0}}=3.9 \mathrm{MeV} / \mathrm{c}^{2}$ for 2004 and $\sigma_{\pi \gamma \gamma}=5.4 \mathrm{MeV} / \mathrm{c}^{2}$ and $\sigma_{\pi \pi^{0}}=3.3 \mathrm{MeV} / \mathrm{c}^{2}$ for 2007 data. 

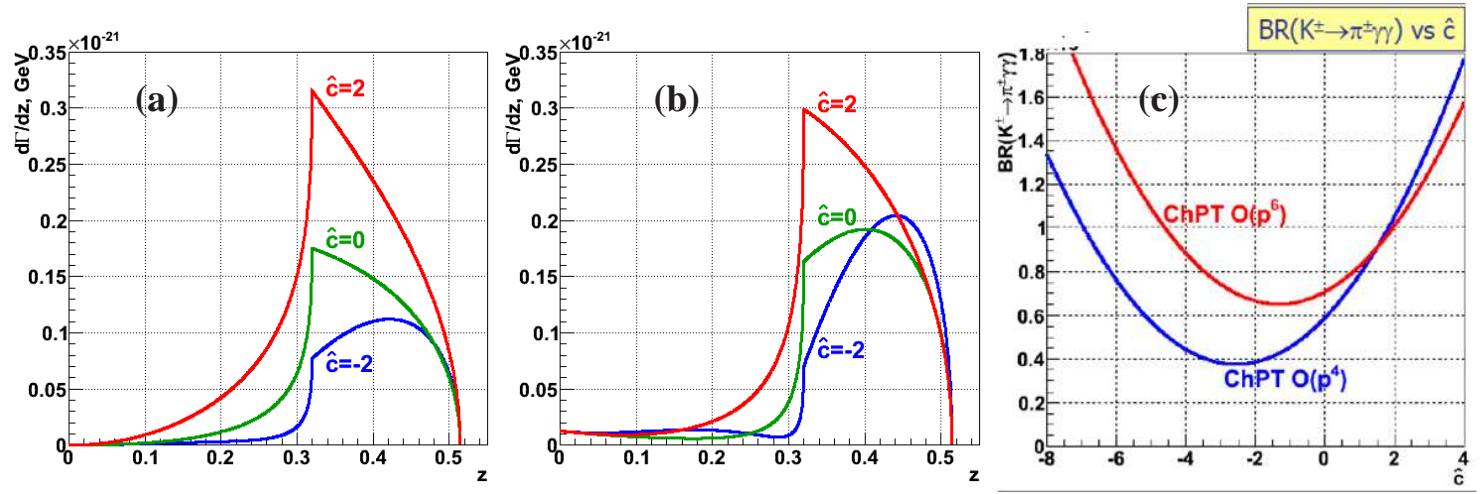

Figure 1: Differential rate $d \Gamma / d z$ vs z for $O(p 4)(a)$ and $O(p 6)(b)$. (c) Branching ratio as a function of $\hat{c}$

\begin{tabular}{|l|c|c|}
\hline Channel & 2004 data & 2007 data \\
\hline $\mathrm{K}^{ \pm} \rightarrow \pi^{ \pm} \gamma \gamma$ candidates & 147 & 175 \\
\hline$\pi^{ \pm} \pi^{0}(\gamma)$ bkg & $11.0 \pm 0.8$ & $11.1 \pm 1.0$ \\
\hline $3 \pi \mathrm{bkg}$ & $5.9 \pm 0.7$ & $1.3 \pm 0.3$ \\
\hline $\mathrm{K}^{ \pm} \rightarrow \pi^{ \pm} \gamma \gamma$ signal & $130 \pm 12$ & $163 \pm 13$ \\
\hline Normalization & $35.731 \cdot 10^{6}$ & $45.034 \cdot 10^{6}$ \\
\hline
\end{tabular}

Table 1: Statistics accumulated in both 2004 and 2007 run

\begin{tabular}{|l|c|c|c|}
\hline & 2004 data & 2007 data & 2004 and 2007 combined \\
\hline $\mathrm{O}\left(p^{4}\right)$ fit & $1.36 \pm 0.33_{\text {stat }} \pm 0.07_{\text {syst }}$ & $1.71 \pm 0.29_{\text {stat }} \pm 0.06_{\text {syst }}$ & $1.56 \pm 0.22_{\text {stat }} \pm 0.07_{\text {syst }}$ \\
\hline $\mathrm{O}\left(p^{6}\right)$ fit & $1.67 \pm 0.39_{\text {stat }} \pm 0.09_{\text {syst }}$ & $2.21 \pm 0.31_{\text {stat }} \pm 0.08_{\text {syst }}$ & $2.00 \pm 0.24_{\text {stat }} \pm 0.09_{\text {syst }}$ \\
\hline Branching ratio & $(0.94 \pm 0.08) \cdot 10^{-6}$ & $(1.06 \pm 0.07) \cdot 10^{-6}$ & $(1.01 \pm 0.06) \cdot 10^{-6}$ \\
\hline
\end{tabular}

Table 2: Results for $\hat{c}$ fits and for the branching ratio

Table 1 shows the number of candidate events and the background for the two data sets. Figure 2-a displays the mass distribution of the events from the 2004 data, together with the Montecarlo expectation of the signal and the major background channels. Figure 2-b is the plot of the same variable as a function of $\mathrm{z}$. Analogously, figures 3-a and 3-b show the same distributions for the 2007 data. Table 2 gives the results of the fits to $\mathrm{O}\left(p^{4}\right)$ and $\mathrm{O}\left(p^{6}\right)$ parametrizations.

The model dependent branching ratio has been measured, assuming the $\mathrm{O}\left(\mathrm{p}^{6}\right)$ prediction and using $\hat{c}$ directly measured from our data, integrating over all the $\mathrm{z}$ range. The preliminary result is $B R\left(\mathrm{~K}^{ \pm} \rightarrow \pi^{ \pm} \gamma \gamma\right)=(1.01 \pm 0.06) \cdot 10^{-6}$, from the combined analyses, including correlated systematics (Fig. 4). This is a factor 5 improvement with respect to the current PDG value $B R\left(\mathrm{~K}^{ \pm} \rightarrow \pi^{ \pm} \gamma \gamma\right)=(1.10 \pm 0.32) \cdot 10^{-6}$ The distributions in $\mathrm{z}$ show clearly the cusp structure, however the results of the fit cannot discriminate between the two ChPT predictions and the analysis cut on z doesn't allow the exploration of the low z region where the enhancement due to $\mathrm{O}\left(p^{6}\right)$ should appear.

The analysis will be soon completed with the measurement of the model independent BR measurement (for $\mathrm{z}>0.2$ ) and with the more accurate extraction of $\hat{c}$ from a fit to $\mathrm{M}_{\gamma \gamma}$ and to the 

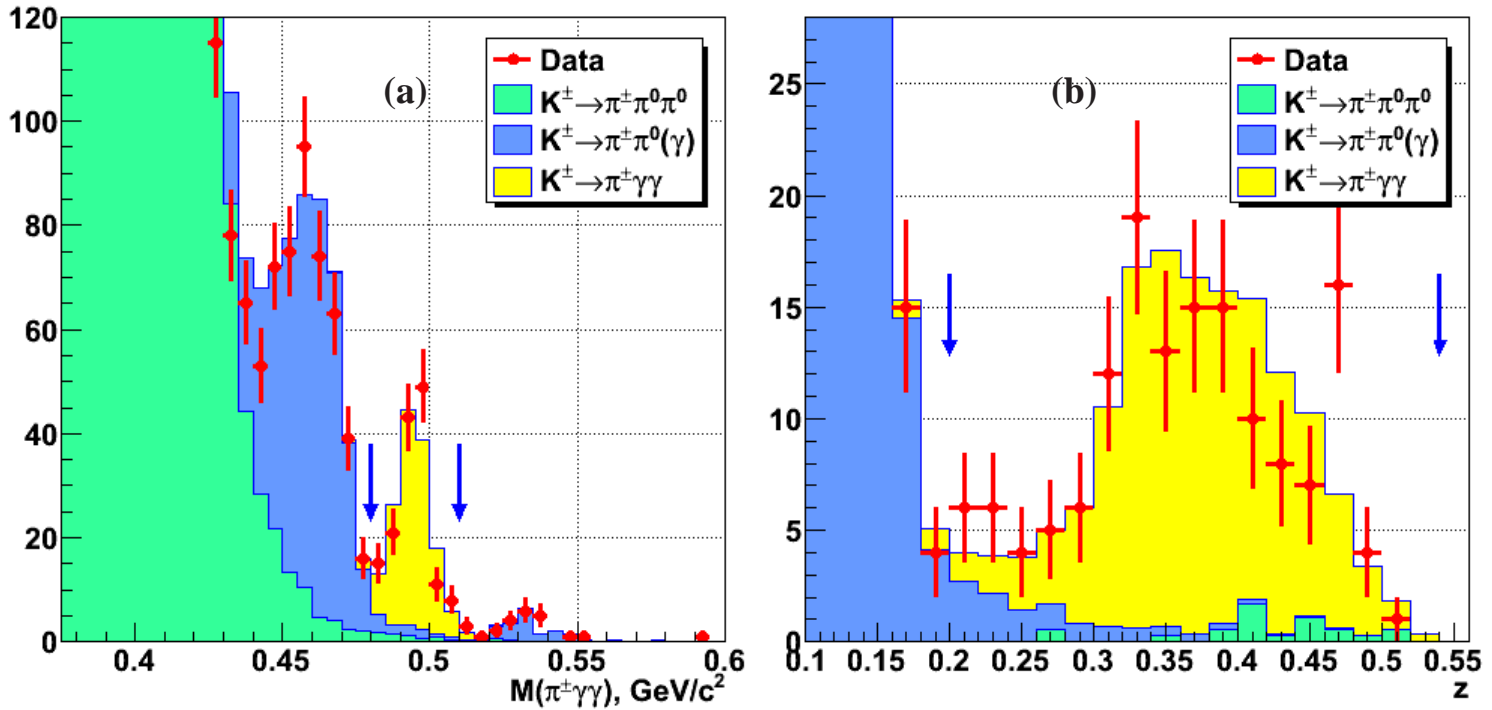

Figure 2: (a) Reconstructed $\pi \gamma \gamma$ mass and (b) z distribution for 2004 data. - Arrows show the applied cuts
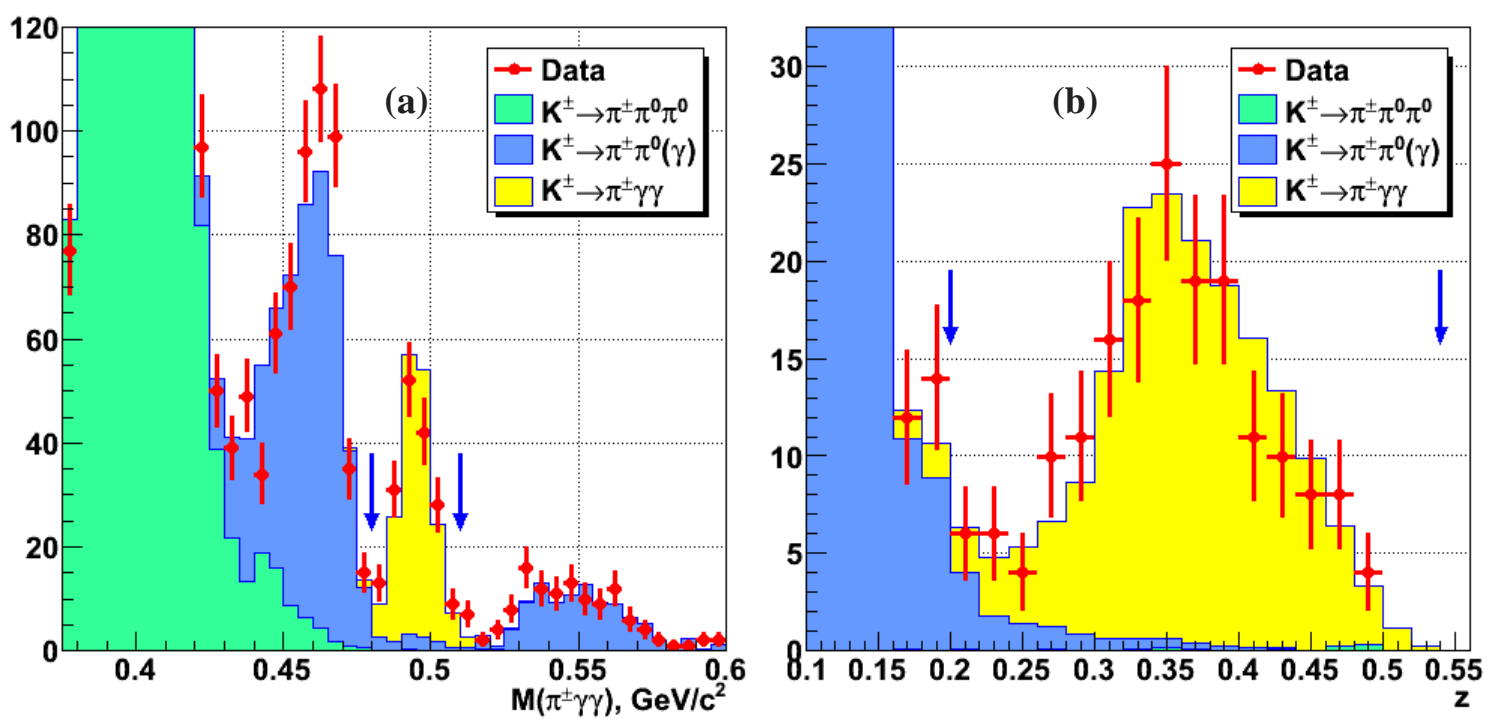

Figure 3: (a) Reconstructed $\pi \gamma \gamma$ mass and (b) z distribution for 2007 data. - Arrows show the applied cuts

BR.

\section{$5.2 \mathrm{~K}^{ \pm} \rightarrow e v \gamma$}

The amplitude of the decay $\mathrm{K}^{ \pm} \rightarrow l^{ \pm} v \gamma$ includes Inner Bremsstrahlung (IB) and Structure Dependent (SD) contributions. The IB part is purely electromagnetic and can be predicted to the lowest order on general grounds. The SD part is sensitive to the kaon structure, in particular the effective coupling to the photon. This coupling could be parametrized in terms of vector $\left(F_{V}\left(p^{2}\right)\right)$ and axial-vector $\left(F_{A}\left(p^{2}\right)\right)$. Predictions for $F_{V}$ and $F_{A}$ exist from ChPT up to $\mathrm{O}\left(p^{6}\right)$ and from specific models (Light front quark model, ChPT with VMD, constituents quark model). The analysis of this decay allows a comparison of these predictions. A recent measurement from KLOE [7], using 1484 events in the kinematical range of $10 \mathrm{MeV}<E_{\gamma}^{*}<250 \mathrm{MeV}$ and $P_{e}^{*}>200 \mathrm{MeV} / \mathrm{c}$, gives a 


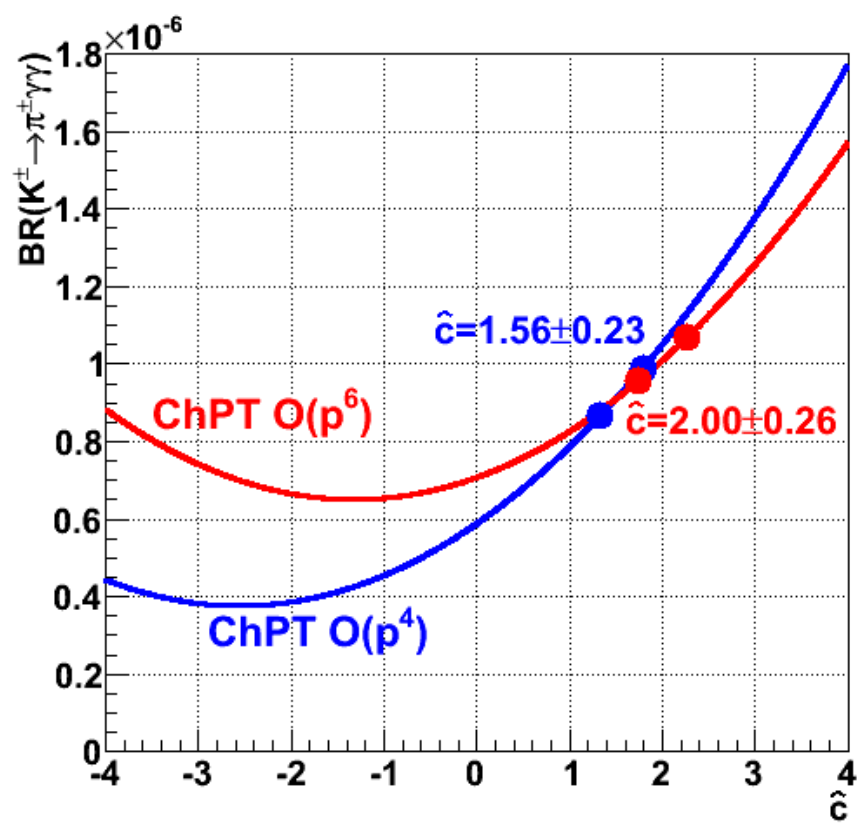

Figure 4: Branching ratio vs $\hat{c}$ with the results of the fit superimposed

branching ratio of $(9.4 \pm 0.4) \cdot 10^{-6}$. The NA62 experiment is expecting to have 10 time more events, despite the reduced kinematical region $\left(50 \mathrm{MeV}<E_{\gamma}^{*}<250 \mathrm{MeV}\right.$ and $\left.P_{e}^{*}>235 \mathrm{MeV} / \mathrm{c}\right)$, with a potential for a model independent extraction of form factors, a validation of the various models and a measurement of ChPT parameters with unprecedented precision.

The results presented here are based on $40 \%$ of the statistics, for a total of about 10000 events, considering only the favoured $\mathrm{SD}^{+}$component (positive photon helicity). The acceptance is $7 \%$ and the background contamination is about $5 \%$. The major contributions to the background come from

- $\mathrm{K}^{ \pm} \rightarrow e^{ \pm} v \pi^{0}$ with $\pi^{0} \rightarrow \gamma \gamma$ and one $\gamma$ is lost.

- $\mathrm{K}^{ \pm} \rightarrow \pi^{ \pm} \pi^{0}$ with $\pi^{0} \rightarrow \gamma \gamma$ : one $\gamma$ is lost and the $\pi^{ \pm}$is misidentified as an $e^{ \pm}$.

- $\mathrm{K}^{ \pm} \rightarrow e^{ \pm} v \gamma(\mathrm{IB})$, the same final state, but with low values of $E_{\gamma}^{*}$, equivalent to low values of $x=2 E_{\gamma}^{*} / M_{K}^{2}$.

The systematic error is expected to be dominated by the background subtraction.

Fig. 5-a shows the plot of the missing mass to $e \gamma$, comparing data and the simulation contributions of data and backgrounds. The non perfect agreement on the left-hand side is due to the effect of non gaussian tails in the spectrometer which will be improved in the next phases of the analysis.

Fig. 5-b shows the $\mathrm{x}$-distribution of the data and the simulation (assuming $\mathrm{ChPT}$ at $\mathrm{O}\left(p^{6}\right)$ ) and the $\chi^{2}$ of the fit. This preliminary result is obtained with the richer $K^{+}$sample. The full analysis will use also the $K^{-}$sample, where the background is expected to be at a higher level and whose analysis will need a dedicated tuning. 

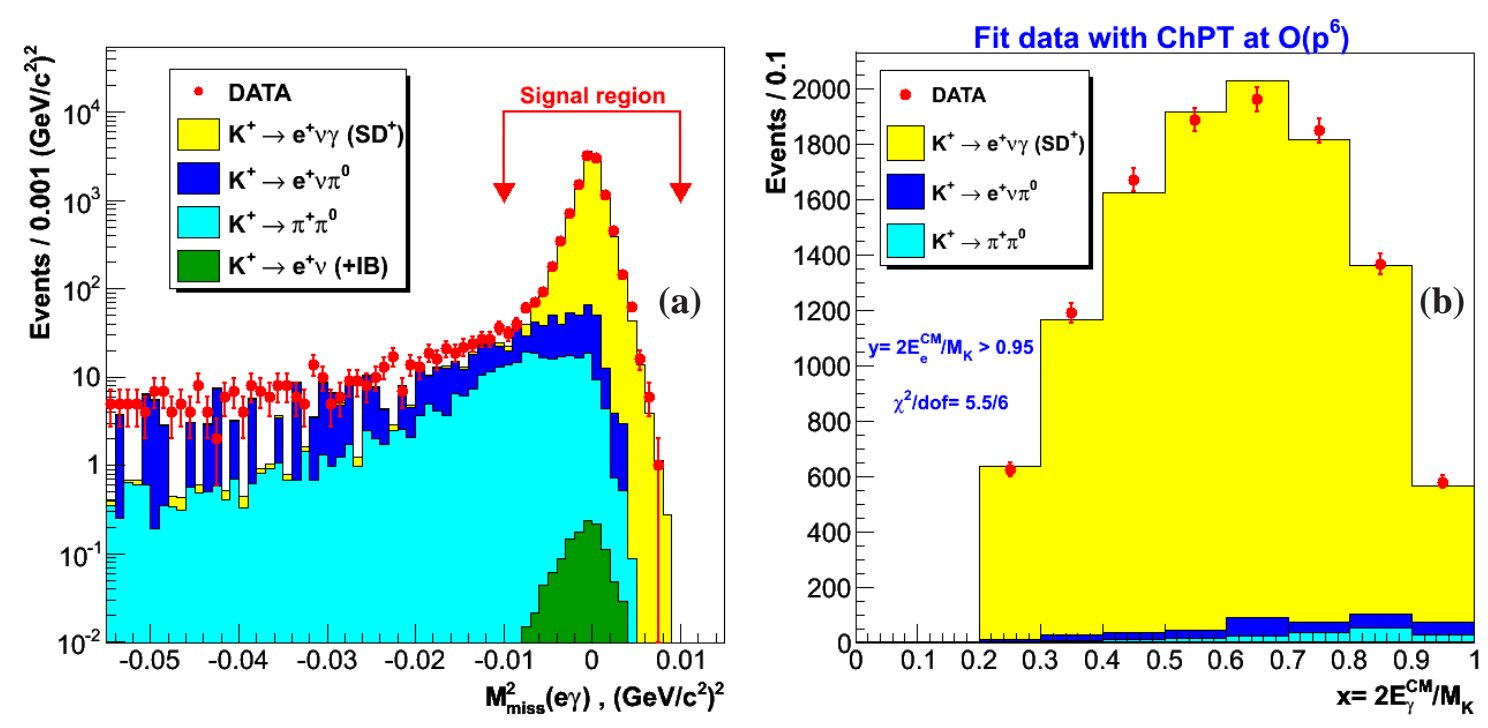

Figure 5: (a) Missing mass to the e $\gamma$ system. (b) x distribution.

\section{$5.3 \mathrm{~K}^{ \pm} \rightarrow \pi^{ \pm} \pi^{0} e^{+} e^{-}$}

The decay $\mathrm{K}^{ \pm} \rightarrow \pi^{ \pm} \pi^{0} e^{+} e^{-}$is dominated by long-distance effects, like radiative kaon decays, with the larger contribution by one-photon exchange, i.e., $\mathrm{K}^{ \pm} \rightarrow \pi^{ \pm} \pi^{0} \gamma^{*} \rightarrow \mathrm{K}^{ \pm} \rightarrow \pi^{ \pm} \pi^{0} e^{+} e^{-}$[8]. Its interest is, besides a possible test of ChPT from the measurement of electric and magnetic form factors, the appearance of short-distance effects by looking into P-violation in the lepton pair system. Contrary to $\mathrm{K}^{ \pm} \rightarrow \pi^{ \pm} \pi^{0} \gamma^{*}$, there is no need to study charge asymmetries. In addition, while $K_{L} \rightarrow \pi^{+} \pi^{-} e^{+} e^{-}$receives $\varepsilon$-type contributions, those are not present in $\mathrm{K}^{ \pm} \rightarrow \pi^{ \pm} \pi^{0} e^{+} e^{-}$ and a CP-violating signal in the charged decay is a genuine short-distance effect, 1) coming from $\mathrm{K}^{ \pm} \rightarrow \pi^{ \pm} \pi^{0} \gamma^{*}$ and 2) associated with effective dimension-6 operators, where one can have also contribution from physics beyond the SM.

Data from NA48/2 are currently being analyzed. A preliminary analysis gives about 4500 candidate events, well above the background. Figure 6 shows the preliminary reconstructed mass distribution of signal and background; the arrows show the applied cuts.

\section{Conclusion}

The NA48/2 and NA62 experiments have used the huge statistics accumulated in 2004 and 2007 to study many rare charged kaon decays, testing Chiral perturbation theory predictions. We have described here the results of the analysis of the decay $\mathrm{K}^{ \pm} \rightarrow \pi^{ \pm} \gamma \gamma$ and a preliminary comparison of the $\mathrm{K}^{ \pm} \rightarrow \mathrm{e}^{ \pm} v \gamma$ decay with the $\mathrm{O}\left(p^{6}\right)$ prediction. The first observation of the decay $\mathrm{K}^{ \pm} \rightarrow \pi^{ \pm} \pi^{0} e^{+} e^{-}$has been reported.

\section{References}

[1] J.R. Batley et al., Eur. Phys. J. C52 (2007) 875. 


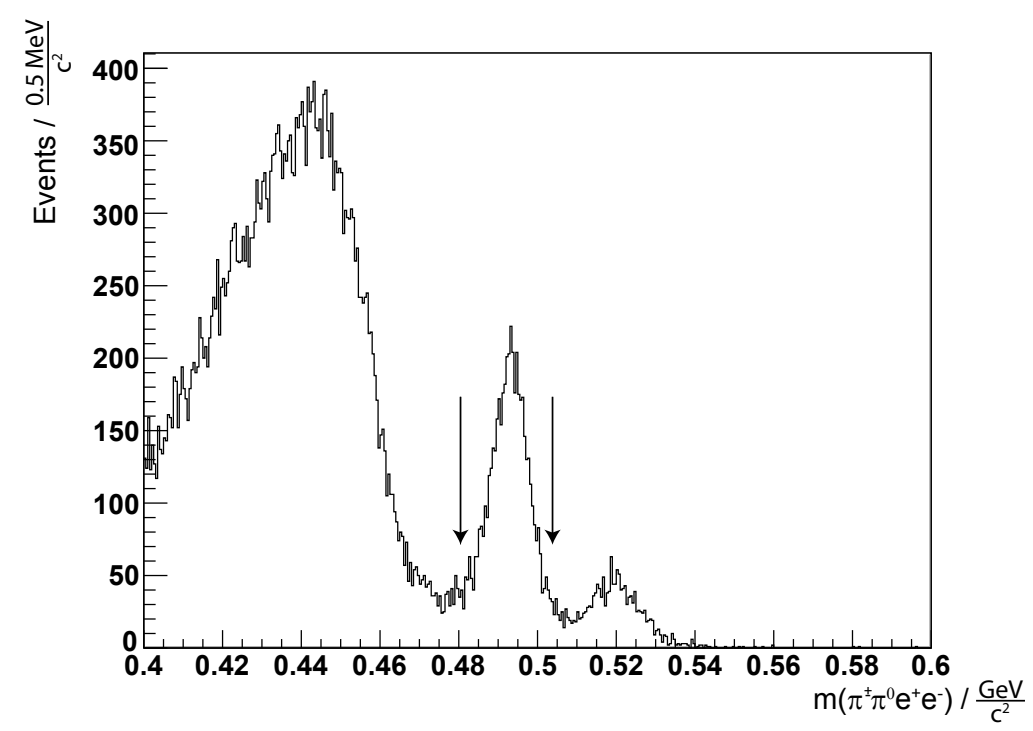

Figure 6: Mass spectrum of the reconstructed decay $\mathrm{K}^{ \pm} \rightarrow \pi^{ \pm} \pi^{0} e^{+} e^{-}$

[2] S. Weinberg, Physica $A 96$ (1979) 327; J. Gasser and H. Leutwyler, Ann. Phys. (N.Y.) 158 (1984) 142; A.V. Manohar and H. Georgi, Nucl. Phys. B234 (1984) 189; G. D’Ambrosio and G. Isidori, Int. J. Mod. Phys. A13 (1998) 1.

[3] C. Lazzeroni, Phys. Lett. B698 (2011) 105.

[4] V. Fanti et al., Nucl. Inst. Methods A574 (2007) 433.

[5] G. D’Ambrosio and J. Portoles, Phys. Lett. B386 (1996) 403, Phys. Lett. B389 (1996) 770, erratum ibid. B395 (1997) 390.

[6] P. Kitching et al., Phys. Rev. Lett. 79 (1997) 4079.

[7] F. Ambrosino et al., Eur. Phys. J., C64 (2009) 627, erratum ibid. C65 (2010) 703.

[8] L. Cappiello, O. Catà, G. D’Ambrosio and Dao-Neng Gao, Eur. Phys. J., C72 (2012) 1872. 